\title{
An archaeal origin of the Wood-Ljungdahl H4MPT branch and the emergence of bacterial methylotrophy
}

Authors: Panagiotis S. Adam ${ }^{1,2, \dagger}$, Guillaume Borrel $^{1}$, Simonetta Gribaldo ${ }^{1 *}$

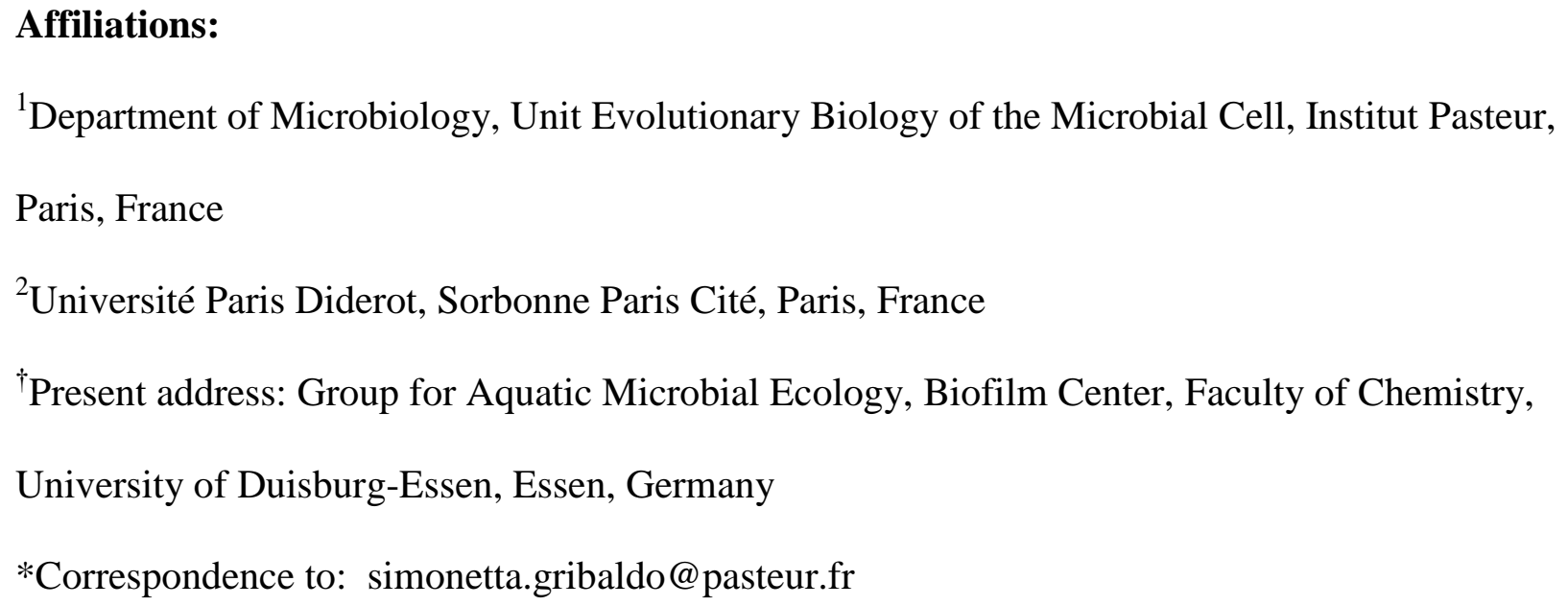

The tetrahydromethanopterin $\left(\mathrm{H}_{4} \mathrm{MPT}\right)$ methyl branch of the Wood-Ljungdahl (WL) pathway is shared by archaeal and bacterial metabolisms that greatly contribute to the global carbon budget and greenhouse gas fluxes: methanogenesis and methylotrophy, including methanotrophy ${ }^{1-3}$. It has been proposed that the $\mathrm{H}_{4} \mathrm{MPT}$ branch dates back to the Last Universal Common Ancestor $(\text { LUCA })^{4-6}$. Interestingly, it has been recently identified in a number of recently sequenced and mostly uncultured non-methanogenic and non-methylotrophic archaeal and bacterial lineages, where its function remains unclear ${ }^{5,7}$ Here, we have examined the distribution and phylogeny of the enzymes involved in the $\mathrm{H}_{4} \mathrm{MPT}$ branch and the biosynthesis of its cofactors in over 6400 archaeal and bacterial genomes. We find that a full WL-H4 4 MPT pathway is widespread in Archaea and likely ancestral to this domain, whereas this is not the case for Bacteria. Moreover, the inclusion 
of recently sequenced lineages leads to an important shortening of the branch separating Archaea and Bacteria with respect to previous phylogenies of the $\mathrm{H}_{4} \mathrm{MPT}$ branch. Finally, the genes for the pathway are co-localized in many of the recently sequenced archaeal lineages, similar to bacteria. Together, these results weaken the LUCA hypothesis and rather favor an origin of the $\mathrm{H}_{4} \mathrm{MPT}$ branch in Archaea and its subsequent transfer to Bacteria. We propose a scenario for its potential initial role in the first bacterial recipients, and its evolution up to the emergence of aerobic methylotrophy. Finally, we discuss how an ancient horizontal transfer not only triggered the emergence of key metabolic processes but also important transitions in Earth's history.

The WL pathway consists of the reversible reduction of $\mathrm{CO}_{2}$ into the carbonyl and methyl moieties of acetyl-CoA through two separate (carbonyl and methyl) branches ${ }^{8}$ (Figure 1). There exist two versions of the methyl branch, one using tetrahydrofolate $\left(\mathrm{H}_{4} \mathrm{~F}\right)$, and the other methanofuran (MF) and tetrahydromethanopterin $\left(\mathrm{H}_{4} \mathrm{MPT}\right)$ as cofactor carriers of reduced carbon. Archaea use the $\mathrm{H}_{4} \mathrm{MPT}$ methyl branch either in the reductive direction for carbon fixation and/or $\mathrm{CO}_{2}$-reducing methanogenesis, or in reverse for anaerobic acetyl-CoA or methane oxidation $^{7}$. Other uses have been proposed, such as participation in the oxidation of short-chain alkanes ${ }^{3,9}$. Five enzymatic steps are involved: in the reductive direction, (1) $\mathrm{CO}_{2}$ is reduced to MF-bound formyl (Fwd enzyme complex), (2) formyl is transferred to $\mathrm{H}_{4} \mathrm{MPT}$ (Ftr), cyclized into (3) methenyl (Mch), and then progressively reduced to (4) methylene (Mtd/Hmd) and (5) methyl (Mer) (Figure 1). Then, the $\mathrm{H}_{4}$ MPT-bound methyl is either transferred to coenzyme M and reduced to methane (methanogenesis), or combined with $\mathrm{CO}$ and $\mathrm{CoA}$ to form acetyl-CoA in the carbonyl branch of the WL pathway $\left(\mathrm{CO}_{2}\right.$ fixation). In methylotrophic Proteobacteria, the $\mathrm{H}_{4} \mathrm{MPT}$ branch is entered at methylene- $\mathrm{H}_{4} \mathrm{MPT}$ through the formaldehyde-activating enzyme 
(Fae) and operates in the oxidative direction (via bMtd and Mch) before being diverted into the $\mathrm{H}_{4} \mathrm{~F}$ methyl branch of the WL pathway (Fhc), the Serine cycle, and the Calvin cycle (Figure 1). The $\mathrm{H}_{4} \mathrm{MPT}$ branch appears as a module in aerobic methylotrophy or as standalone thus playing roles in carbon assimilation, formaldehyde detoxification, and energy conservation ${ }^{10}$. The initial discovery of the $\mathrm{H}_{4}$ MPT branch in methylotrophic Proteobacteria 20 years ago gave rise to two alternative hypotheses on its evolutionary origin: either it was acquired via horizontal transfer from methanogenic archaea or it was inherited from the Last Universal Common Ancestor (LUCA) ${ }^{11,12}$. The LUCA scenario gained traction when the $\mathrm{H}_{4} \mathrm{MPT}$ branch was also found in Planctomycetes, where it is supposed to perform formaldehyde oxidation ${ }^{4}$. Two main arguments were put forward: (i) the distinct separation between Bacteria and Archaea in phylogenies of some $\mathrm{H}_{4}$ MPT components (Figure 2a) and cofactor biosynthesis genes, and (ii) the lack of gene co-localization in archaeal methanogens as opposed to Bacteria, making a concurrent transfer inherently difficult ${ }^{4,13,14}$. However, considering the large number of independent losses of the pathway in Bacteria implied by the LUCA hypothesis, the transfer scenario has remained a valid alternative ${ }^{13}$.

The presence of an $\mathrm{H}_{4} \mathrm{MPT}$ branch has since been highlighted in members of several bacterial lineages. These include some Gemmatimonadetes, proposed to be aerobic methylotrophs ${ }^{15}$, and the candidate division NC10, including Candidatus Methylomirabilis oxyfera which is anaerobic and performs methane oxidation coupled to denitrification ${ }^{16}$. Nonetheless, many bacteria with an $\mathrm{H}_{4} \mathrm{MPT}$ branch are non-methylotrophic and their use of the pathway is unknown ${ }^{2,5}$. Even among Proteobacteria there exist some non-methylotrophs (e.g. members of the Burkholderiales) which probably use the branch for formaldehyde oxidation ${ }^{10}$. The $\mathrm{H}_{4} \mathrm{MPT}$ branch has also been recently found in a number of uncultured, non-methanogenic archaea, including Persephonearchaea 
(MSBL1), Hadesarchaea, Theionarchaea, Altiarchaeales, Thorarchaeota, Bathyarchaeota, and Lokiarchaeota $^{9}$. This wider distribution has been proposed to support the LUCA scenario ${ }^{2,5}$. Moreover, a recent survey of genes potentially present in the LUCA found some components of the $\mathrm{H}_{4}$ MPT methyl branch, although not the complete pathway ${ }^{6}$. However, a comprehensive phylogenetic analysis including all the recently discovered lineages has been lacking, and the evolution of several $\mathrm{H}_{4} \mathrm{MPT}$ and MF biosynthetic genes has never been studied. By screening a local databank of 643 archaeal and 5,796 bacterial genomes, we could infer the presence of a complete or almost complete $\mathrm{H}_{4} \mathrm{MPT}$ methyl branch in the majority of analyzed archaeal lineages, while its distribution in Bacteria appears to be much more restricted (Supplementary Data 1). We found bacterial homologs of the Fwd complex, Ftr, and Mch, but no homologs of Mtd. Concerning Mer, it is a member of the vast luciferase-like family, and we could not identify it from our homology searches. However, it has never been reported in Bacteria. Although most single gene phylogenies could not be completely resolved because of poor sequence conservation, a few intradomain transfers, and the presence of paralogous copies (Supplementary Figures 1-7), three subunits of the Fwd complex (FwdABC) had a congruent history and could be concatenated to increase their phylogenetic signal (Methods). The FwdABC phylogeny is overall resolved (Figure $2 \mathrm{~b}$ and Supplementary Figure 8). Interestingly, the addition of the recently sequenced lineages leads to a shortening of the branch separating Archaea and Bacteria to one tenth and one fifth with respect to that obtained using lineages that were available in $1999^{12}$ and $2004^{4,13}$ respectively (Figure 2a). Such a short branch is unlike those usually observed for genes inferred in the LUCA (see for example ${ }^{17}$ ). Similar results were obtained for Mch (1999: 0.8941, 2004: 0.3426, this study: 0.0793 substitutions per site, respectively). Strikingly, the genes for the $\mathrm{H}_{4}$ MPT branch are often co-localized in the newly added archaeal 
lineages (Figure 2c), consistently with recent observations ${ }^{2}$. This is unlike methanogens but similar to Bacteria, including the non-methylotrophs (Figure 2c). These results (evolutionary proximity of archaeal and bacterial homologs and gene co-localization in archaea) weaken the two main arguments previously put forward in favor of the LUCA scenario, and rather support a horizontal transfer.

Regarding the direction of the transfer, a number of arguments favor one from Archaea to Bacteria. The archaeal part of the FwdABC phylogeny is overall consistent with the known relationships within this domain ${ }^{18}$, in particular the presence of clades corresponding to the Euryarchaeota and TACK-Asgard and the monophyly of major lineages within (Figure 2b). Despite being less resolved and containing a few horizontal gene transfers, the phylogenies of the other components of the pathway (Ftr, Mch, Mtd, Mer) also show overall similar clades, suggestive of a similar evolutionary history (Supplementary Figures 1, 2, 4 and 6, and their legends for additional discussion). Coupled with the wide taxonomic distribution of the $\mathrm{H}_{4} \mathrm{MPT}$ methyl branch in Archaea (Supplementary Data 1), this strongly suggests its presence in the Last Archaeal Common Ancestor (LACA). In addition, almost all archaeal lineages that possess the $\mathrm{H}_{4} \mathrm{MPT}$ branch also have the carbonyl branch (Supplementary Data 1), granting them a complete WL pathway. In contrast, the two branches never coexist in Bacteria, with the exception of some Firmicutes and one $\delta$-Proteobacteria which are homoacetogens/acetate oxidizers ${ }^{19,20}$ (Supplementary Data 1). Because a functional carbonyl branch was likely present in the LUCA, and therefore in the ancestor of all Bacteria ${ }^{21}$, this lack of co-occurrence could be explained by the fact that the $\mathrm{H}_{4}$ MPT branch in Bacteria was indeed acquired later from Archaea. Under any of the current three alternative rootings of the archaeal phylogeny (i.e. in between Euryarchaeota and the TACK, at the DPANN, or within Euryarchaeota; see ${ }^{18}$ and references 
therein), the bacterial homologs stem from within the archaeal radiation, and in particular close to the Altiarchaeales, Hadesarchaea, Persephonearchaea (MSBL1), and Theionarchaea (Figure 2b). The hypothesis of a transfer from Archaea to Bacteria is also supported by the overall similar topologies of the Ftr and Mch trees (Supplementary Figures 1 and 2) to the FwdABC tree.

An argument against the transfer scenario could be the fact that until recently, Archaea were known to use enzymes non-homologous to the bacterial ones for the methylene-methenyl oxidoreduction (Mtd and Hmd). Surprisingly though, several members of the recently sequenced archaeal lineages analyzed here possess a bMtd homolog together with or instead of Mtd (Supplementary Data 1). Also, these bMtd homologs are commonly clustered in both archaeal and bacterial genomes with the other $\mathrm{H}_{4}$ MPT branch genes (Figure $2 \mathrm{c}$ ). Although the bMtd phylogeny is poorly resolved (Supplementary Figure 3), these results suggest that bMtd was also likely part of the original transfer from Archaea to Bacteria.

Under the hypothesis that the $\mathrm{H}_{4} \mathrm{MPT}$ branch was transferred from Archaea to Bacteria, it is expected that this event would have involved also enzymes necessary for the biosynthesis of the $\mathrm{MF}$ and $\mathrm{H}_{4} \mathrm{MPT}$ cofactors. It should be mentioned that many steps of folate and $\mathrm{H}_{4} \mathrm{MPT}$ biosynthesis are catalyzed by different enzymes across Bacteria and Archaea. Some of the genes involved in $\mathrm{H}_{4} \mathrm{MPT}$ cofactors biosynthesis in Archaea (e.g. MptD, MptE) are present in a few bacteria but generally not in the ones that possess the H4MPT branch ${ }^{22}$, and thus they were omitted from our analysis.

Along with the discovery of the $\mathrm{H}_{4} \mathrm{MPT}$ branch in Planctomycetes, it was observed that in Bacteria these genes are clustered with some genes related to methanol oxidation and to the biosynthesis of pterin cofactors ${ }^{4,13,14,23}$. Two genes encode dihydromethanopterin reductase 
(DmrX/DmrB/AfpA) and $\beta$-ribofuranosylaminobenzene 5'-phosphate synthase (MptG). Ten other genes had unknown or only predicted functions and were referred to as numbered open reading frames $(\mathrm{ORFs})^{4,13,14,23}$. The phylogenies of some of these ORFs and MptG were found to be similar to those of the $\mathrm{H}_{4}$ MPT branch components ${ }^{4,13,14}$, suggesting a common evolutionary history. Mutagenesis experiments and bioinformatic annotation in Methylobacterium extorquens suggested a role in $\mathrm{H}_{4} \mathrm{MPT}$ biosynthesis for most of these $\mathrm{ORFs}^{14,23}$. Since then, the genes for MF and to some extent $\mathrm{H}_{4}$ MPT cofactor biosynthesis have been characterized in the archaeon Methanocaldococcus jannaschii and other methanogens, and their correspondence to the $M$. extorquens ORFs has been assigned (see Supplementary Figures 9-10 and their legends for additional discussion and references).

Similar to the $\mathrm{H}_{4} \mathrm{MPT}$ branch, we analyzed the distribution and phylogeny of these twelve genes (10 ORFs, DmrX/DmrB/AfpA, MptG) in our archaeal and bacterial genomes (Supplementary Data 1 and Supplementary Figures 11-20). Seven of the original ORFs correspond to the characterized enzymes in the MF (Orf1/MfnD, Orf9/MfnF, Orf21/MnfE, Orf22/MnfB) and putatively in the $\mathrm{H}_{4} \mathrm{MPT}$ (Orf5/MptN, Orf20, OrfY) biosynthesis pathways (Figure 2c, Supplementary Figures 9-10 and their legends for additional discussion and references). Moreover, we could putatively assign two of the remaining ORFs $($ Orf7, 17) to yet uncharacterized reactions of $\mathrm{H}_{4} \mathrm{MPT}$ biosynthesis, allowing us to propose an almost complete pathway (Figure 2c, Supplementary Figure 10 and its legend for additional discussion and references).

Despite the lack of resolution and the presence of horizontal transfers, the phylogenies of these enzymes (Supplementary Figures 11-20) are very similar to those of the $\mathrm{H}_{4} \mathrm{MPT}$ branch. This suggests that the same horizontal transfer from Archaea might have introduced the $\mathrm{H}_{4} \mathrm{MPT}$ 
branch in bacteria along with the necessary cofactor biosynthesis enzymes, an event that would have involved at least 18 genes. This scenario is also supported by co-localization of cofactor biosynthesis and $\mathrm{H}_{4} \mathrm{MPT}$ branch genes in both Bacteria and Archaea (Figure 2c).

Interestingly, most cofactor biosynthesis genes have a wide distribution in Archaea with respect to Bacteria (Supplementary Data 1). Their presence in various Crenarchaeota and -for some- in Thermococcales has been known ${ }^{4,13}$ but we also found homologs in Heimdallarchaeota and Aigarchaeota (Supplementary Data 1). These genes might represent functionally-repurposed remnants of an ancestral $\mathrm{H}_{4}$ MPT branch which was lost in these lineages.

It has been proposed that bacterial methylotrophy likely arose from the combination of different modules, with the $\mathrm{H}_{4}$ MPT branch predating it ${ }^{2,10}$. The inclusion of current bacterial and archaeal diversity in our analysis allows us to draw a more comprehensive scenario for the assembly of methylotrophy around the $\mathrm{H}_{4} \mathrm{MPT}$ branch (Figure 3). From our results, and as discussed above, it is very likely that a complete WL pathway was already present in the LACA (Figure 3, black dot). The $\mathrm{H}_{4} \mathrm{MPT}$ branch (Fwd, Ftr, Mch, and possibly bMtd) plus the cofactor biosynthesis enzymes (but not the carbonyl branch) were then transferred to Bacteria (Figure 3, red dot), and then spread through a combination of vertical inheritance followed by multiple independent losses and further intra-domain transfers. Planctomycetes seem to have acquired at least part of the pathway (Fwd, Ftr) independently two or three times (Figure 2b and Supplementary Figure 8, and Supplementary Figure 1, respectively). A key event was the addition of Fae to the pathway before the divergence of Planctomycetes and the NC10 phylum (Figure 3, green dot). As Fae allows linkage of methyl compound oxidation into formaldehyde to the $\mathrm{H}_{4} \mathrm{MPT}$ branch, this event would have led to the use of the pathway for formaldehyde oxidation and eventually for (denitrifying) methylotrophy in NC10. There exist multiple Fae homologs (Fae-like proteins) in 
Bacteria (Supplementary Data 1), but the canonical Fae is likely the original component, as it colocalizes with the $\mathrm{H}_{4}$ MPT genes (Figure 2c), is widespread in methylotrophs (Supplementary Data 1), and is the only one whose mutant cannot be complemented by other homologs ${ }^{4,14}$. The Fae homologs in bacteria might also have originated through subsequent transfers from Archaea, where the enzyme is far more widespread (Supplementary Data 1 and Supplementary Figure 7). At this point, methylene- $\mathrm{H}_{4} \mathrm{MPT}$ was still fully oxidized to $\mathrm{CO}_{2}$. The next milestone would have been the loss of the FwdD subunit (Figure 3, orange dot). In organisms without FwdD, like $\alpha, \beta, \gamma-$ Proteobacteria and most Planctomycetes, the FwdABC subunits and Ftr form a complex called $\mathrm{Fhc}^{13,24}$ (Figure 1). Fhc performs the formyl transfer to MF analogs and then lyses it to formate instead of oxidizing it fully to $\mathrm{CO}_{2}$, since it probably lacks the formate dehydrogenase activity of the FwdBD subcomplex ${ }^{25}$. Formate fluxes could be diverted to $\mathrm{CO}_{2}$ (using Fdh) and to methylene- $\mathrm{H}_{4} \mathrm{~F}$, to be used in the Serine Cycle or Calvin $\mathrm{Cycle}^{26}$, thus creating the link between the $\mathrm{H}_{4}$ MPT branch and the methylotrophic assimilation pathways (Figure 1), leading to the emergence of contemporary aerobic methylotrophy (Figure 3).

Almost all archaeal lineages that possess the $\mathrm{H}_{4}$ MPT branch also have the carbonyl branch, granting them a complete WL pathway, while this is not the case in Bacteria (Supplementary Data 1). Thus, one major question that remains to be answered is what the potential function of the $\mathrm{H}_{4}$ MPT methyl branch in the pre-methylotrophic (without Fae) bacterial lineages might have been $^{5}$. In the phylogenies of the $\mathrm{H}_{4}$ MPT branch enzymes, the bacterial lineages closest to the putative initial transfer are the Limitata, Synergistetes, and Firmicutes (Clostridia, Halanaerobiales) (Figure 2b and Supplementary Figures 2, 3, 8). 
Limitata are a deep-branching uncultured clade related to Synergistetes (Supplementary Figure 21 ) and are currently represented by two mostly complete (GBS1: $100 \%, 42 \_11: 84.1 \%$ ) metagenome-assembled genomes (MAGs) placed under NCBI's "Unclassified Bacteria". We analyzed the metabolic potential of the Limitata MAGs to gain some clues about the role of the $\mathrm{H}_{4}$ MPT branch (Figure 4, Supplementary Data 2). In Limitata, the $\mathrm{H}_{4}$ MPT branch might potentially work in either the oxidative or the reductive direction. In the reductive direction, the branch could serve as a carbon-fixing electron sink during fermentative growth, as is the case in some Firmicutes ${ }^{27}$. Recently it was also proposed that an uncultured member of the $\delta$ Proteobacteria can autotrophically fix carbon through the $\mathrm{H}_{4} \mathrm{~F}$ branch leading into the Glycine Cleavage System $(\mathrm{GCS})^{28}$. However, given that in other bacteria (Proteobacteria, Planctomycetes, NC10) the $\mathrm{H}_{4} \mathrm{MPT}$ branch operates oxidatively, a reductive direction seems unlikely in Limitata.

In the oxidative direction, methylene is fully oxidized to $\mathrm{CO}_{2}$. In the absence of Fae, though formaldehyde can spontaneously condense with $\mathrm{H}_{4} \mathrm{MPT}$ at low rates ${ }^{29}$, the most plausible way to link methylene- $\mathrm{H}_{4} \mathrm{MPT}$ with the rest of the metabolism (amino acid fermentation, pyruvate from glycolysis) is through serine and glycine (Figure 4). Normally, one methylene- $\mathrm{H}_{4} \mathrm{~F}$ is produced during the conversion of serine to glycine by the serine hydroxylmethyltransferase, and then the GCS converts the glycine to another methylene- $\mathrm{H}_{4} \mathrm{~F}, \mathrm{CO}_{2}$, and $\mathrm{NH}_{3}$. Nevertheless, according to our inference, in Limitata the serine hydroxylmethyltransferase and the GCS should be able to produce methylene- $\mathrm{H}_{4} \mathrm{MPT}$ instead of methylene- $\mathrm{H}_{4} \mathrm{~F}$ (Figure 4). For serine hydroxylmethyltransferase, this indeed seems to occur in Archaea, such as Methanosphaera stadmanae and Methanococcus thermolithotrophicus ${ }^{30}$. The full oxidation process is expected to produce five reducing equivalents per serine molecule, or three per glycine molecule. 
Limitata possess Hox-like and Mbh-like hydrogenases that are possibly used for hydrogen production to regenerate cofactors and dispose of excess reductants resulting from fermentation $^{31}$, including those that would be produced by the $\mathrm{H}_{4} \mathrm{MPT}$ branch working in the oxidative direction $^{31}$ (Figure 4). In lineages with the $\mathrm{H}_{4} \mathrm{MPT}$ branch, such as Halanaerobium (Firmicutes) and Anaerobaculum (Synergistetes, the closest relatives of Limitata)

(Supplementary Data 1), hydrogen production associated to fermentation has also been reported. In these bacteria, presence of the $\mathrm{H}_{4} \mathrm{MPT}$ reactions in addition to normal fermentation might be related to their sizeable biohydrogen yield which is consumed when co-cultured with a methanogen ${ }^{32,33}$. Hydrogen production ties in to the ecological roles of these bacteria: for example Anaerobaculum has been found in oil pipes where it seems to act as a syntrophic partner to a methanogen ${ }^{34}$. Since Limitata genomes originate from environments similar to those of Anaerobaculum and Halanaerobium (oil fields, hot spring sediments), they probably fulfill a similar role. From these considerations, one possibility is that the original role of the $\mathrm{H}_{4} \mathrm{MPT}$ methyl branch in non-methylotrophic Bacteria was to increase their hydrogen production, which in turn would promote $\mathrm{CO}_{2}$ fixation by surrounding bacteria or archaea with a WL pathway and would lead to higher biomass and carbon turnover.

Methane and carbon dioxide, two end products of the WL pathway, represent centerpieces of the global carbon cycle, and have been of crucial importance to global climate change through geological time ${ }^{1,35}$. Having determined the presence of a complete WL pathway in the LACA $\left({ }^{21}\right.$ and this study) lends credibility to previous work that places the origin of methanogenesis at 3.46 Ga or even earlier ${ }^{36}$. In contrast, the origin of methylotrophy is harder to determine. In the past, the extremely ${ }^{13} \mathrm{C}$-depleted (-45 to $-60 \%$ ) isotopic signatures found in the late Archean have been referred to as the "Age of Global Methanotrophy" and attributed over time to aerobic 
methylotrophs, archaeal anaerobic methane oxidizers (ANME), and more recently to multiple uses of the WL pathway, including acetogenesis ${ }^{37}$. However, methylotrophs do not produce carbon isotopic signatures in that range $\mathrm{e}^{38,39}$.

In recent years, the taxonomic range of (bona fide) bacterial methylotrophs has expanded through the discovery of methanol dehydrogenases and the $\mathrm{H}_{4} \mathrm{MPT}$ branch in lineages outside the Proteobacteria, namely Gemmatimonadetes and Acidobacteria ( ${ }^{2,15}$ and this study). This origin of aerobic methylotrophy seems to correspond to the loss of FwdD (Figure 4) and is exemplified by these lineages sometimes being on long branches in our phylogenies, possibly resulting from rapid adaptation (Figure $2 b$, Supplementary Figures 2,11 ). This is especially interesting in the case of $\alpha, \beta, \gamma$-Proteobacteria, as some divergence dating studies have placed their origin close to $2.5 \mathrm{Ga}^{40}$. It could then be speculated that the emergence of aerobic methylotrophy is linked to the Great Oxygenation Event (GOE) and increased oxygen concentrations in the atmosphere. Shortly after the GOE, global temperatures plummeted, causing the Paleoproterozoic glaciations. In some models, this climate shift was driven by the collapse of the methane greenhouse that contributed to sustaining the temperature of the Archean Earth $^{41}$. Various reasons for this collapse have been proposed, such as a strong decrease of methanogen populations due to nickel depletion ${ }^{42}$, and/or an increase in anaerobic methane oxidation spurred by higher sulfate levels ${ }^{43}$. More recently, the contribution of aerobic methanotrophy as a suppressor of methane fluxes slightly prior to the GOE has also been considered $^{44}$. The emergence of aerobic methylotrophy, which includes methanotrophy, near the GOE could have therefore contributed to the collapse of the methane greenhouse. 
In conclusion, our study underlines how an ancient inter-domain transfer of the $\mathrm{H}_{4} \mathrm{MPT}$ methyl branch and its cofactor biosynthesis not only triggered the emergence of key metabolic processes in the carbon cycle but also important transitions in the history of our planet.

\section{Methods}

\section{$\underline{\text { Homology searches }}$}

A local databank of genome sequences from 643 Archaea and 5,796 Bacteria was assembled, representing all genomes (one per species) present at the National Center for Biotechnology Information (NCBI) as of June 2016, and included some retroactively added Bacteria and Archaea recently shown to possess genes of the $\mathrm{H}_{4} \mathrm{MPT}$ pathway, some genomes manually downloaded from the Joint Genome Institute/Integrated Microbial Genomes (JGI/IMG) ${ }^{45}$ and the $\operatorname{ggkbase}^{46}$ (ggkbase.berkeley.edu/). For some genomes with only nucleotide sequences, ORFs were predicted by using Prodigal ${ }^{47}$ with default parameters. A full list of taxa used to build this databank is provided in Supplementary Data 3.

Homology searches for the components of the $\mathrm{H}_{4}$ MPT branch and MF and $\mathrm{H}_{4} \mathrm{MPT}$ biosynthesis genes (FwdA, B, C, D, Ftr, Mch, bMtd, Mtd, Hmd, Fae, Mer, DmrX, MptG, MfnB, D, E, F, OrfY, 5, 7, 17, 19, 20) were performed as follows: from the Kyoto Encyclopedia of Genes and Genomes $(\mathrm{KEGG})^{48}$ orthology page of each enzyme or known homologs from the literature, up to five sequences from both Bacteria and Archaea, preferably characterized, were aligned and used to create preliminary hidden Markov model (HMM) profiles. These were used to perform hidden Markov model-based searches with $\mathrm{HMMer}^{49}$ against our local genome databanks of Bacteria and Archaea separately. The first 20-30 hits in descending e-value order from each domain were used to create a second round of domain-specific HMM profiles for each protein. 
Usually a cutoff of e-4 was applied unless there were too many hits (>>1000) or a very clear demarcation of homologs. In this case, we set the cutoff manually based on the e-value slope and annotations. In some cases when putative homologs (by annotation) were present above the e-4 cutoff, a separate HMM profile based on a larger sampling of sequences was created to pick these divergent homologs. Either way, the resulting datasets were manually refined through preliminary phylogenetic analysis to remove unrelated sequences. Very close homologs from genomes belonging to members of the same species/genus were removed from the final datasets to reduce computational burden. Preliminary Fwd trees were used to distinguish the clades corresponding to characterized tungsten (Fwd), molybdenum (Fmd), and a separate divergent clade referred here as "tertiary" Fwd homologs (containing only BD subunits, see legend to Supplementary Figure 22). Since Fmd and tertiary Fwd exist only in Archaea, only the Fwd homologs were retained for the FwdABC concatenation (FwdD being too restricted in bacteria to be included). The clustering of the genes involved in the $\mathrm{H}_{4} \mathrm{MPT}$ branch in archaeal and bacterial genomes was checked in Genespy ${ }^{50}$ and supplemented using MacSyFinder ${ }^{51}$ and HMM profiles of the related proteins.

\section{$\underline{\text { Phylogenomic analysis }}$}

Single protein datasets were aligned with $\operatorname{MUSCLE}^{52}$ with default parameters and trimmed with BMGE $1.1^{53}$ using the BLOSUM30 substitution matrix to select unambiguously aligned amino acid positions. To check for phylogenetic congruence among FwdA, B, C subunits and among the MfnB, E, F subunits, single gene trees were calculated in IQTree under the TEST option with 100 bootstrap replicates ${ }^{54}$ and collapsed at nodes showing $<80 \%$ bootstrap support. These trees were tested for congruence against a strictly bifurcating tree of a blind concatenation, by 
performing an Internode Certainty (IC) test ${ }^{55}$ in $\operatorname{RaxML}^{56}$. Sequences responsible for incongruences at order level or above (in Archaea: Methanoflorentaceae were treated as part of Methanocellales) or phylum level (in Bacteria: $\alpha, \beta, \gamma$-Proteobacteria were treated as a single phylum, and in Firmicutes the deep-branching order Halanerobiales was treated as separate from Clostridia) were removed and the procedure was repeated until no further incongruence was found. The resulting datasets were then concatenated. The concatenated Fwd dataset (FwdABC, 857 aa) contained no more than $6 \%$ missing taxa per protein, and the concatenated Mfn dataset (MfnBEF, 360 aa) contained no more than 3.4\% missing taxa per protein. An Archaea-only dataset was also created including Fmd and tertiary Fwd for the four main (non-ferredoxin) subunits (FwdABCD). A separate round of congruence testing was performed, which resulted in a concatenated dataset (FwdABCD, 1192 aa) containing no more than $22 \%$ missing taxa per gene. The larger number of missing taxa is due to the tertiary Fwd only containing subunits B and $\mathrm{D}$.

To determine the effect of the addition of novel lineages on the length of the branch separating Archaea and Bacteria, we reconstructed the FwdABC phylogeny using the lineages that were available in $1999^{12}$ (Methanococcales, Methanobacteriales, Methanopyrales, Methanosarcinales, Archaeoglobales, $\alpha, \beta, \gamma$-Proteobacteria), and $2004^{4}$ (same as 1999 plus Planctomycetes) but with all the genomes currently available for them. Phylogenies were calculated in IQTree as described above with 1000 ultrafast bootrstrap replicates ${ }^{57}$.

No concatenation was possible for the other genes (Ftr, Mch, Mtd, Hmd, bMtd, Fae, Mer, DmrX, MptG, MfnD, OrfY, 5, 7, 17, 19, 20). Although their topologies were overall similar, there were some differences due to taxonomic distribution, a few horizontal transfers or poor signal. Phylogenies were inferred in PhyloBayes ${ }^{58}$ under the CAT $+\mathrm{GTR}+\Gamma 4$ model for the Fwd 
concatenations and $\mathrm{LG}+\Gamma 4$ for the single-gene datasets and the MfnBEF concatenation, because of the smaller number of positions, and in IQTree under the TEST option with 100 bootstrap replicates for all datasets. For Bayesian analyses, four independent Markov chain Monte Carlo chains were run until convergence (with the exception of Orf7 that never got to a standard deviation below 0.8 ) and checked by sampling every two cycles with a $25 \%$ burn-in. All phylogenies are technically unrooted but for display purposes we have placed in each case an ad hoc root according to one of the suggested roots of Archaea i.e. between Euryarchaeota and TACK-Asgard or within Euryarchaeota ${ }^{18}$.

For the reference phylogeny of Bacteria, a Bayesian phylogeny was built in PhyloBayes under the $\mathrm{CAT}+\mathrm{GTR}+\Gamma 4$ model using a concatenation of RNA polymerase subunits $\mathrm{B}, \mathrm{B}^{\prime}$, and IF-2 (2,337-aa positions). The tree was rooted according to ${ }^{17}$.

All datasets relative to these analyses (original alignments, trimmed datasets, and trees in Newick format) are provided in Supplementary Data 4.

\section{$\underline{\text { Limitata metabolic annotation }}$}

As with all genomes in our local database for which protein sequences were not available, the nucleotide sequences of bacterium GBS-1 (txid: 1662110) were downloaded and open reading frames were predicted with Prodigal under default settings ${ }^{47}$. The resulting amino-acid sequences were assigned arbitrary accession numbers. Functional annotations for all open reading frames and metabolic processes were predicted using BlastKOALA

(family_eukaryotes+genus_prokaryotes, and species_prokaryotes databases) ${ }^{59}$, supplemented by BLASTp $^{60}$ searches against the non-redundant database at NCBI. Open reading frames were also predicted on the RAST server ${ }^{61}$ with default options, and for those the metabolic annotation was 
done on both RAST and BlastKOALA. The metabolism of the second Limitata representative (bacterium 42_11 (txid: 1635281)) for which amino acid sequences were available, was annotated in the same way. All outputs are included in Supplementary Data 2. Genome completeness and contamination (respectively $100 \%$ and $0 \%$ for GBS-1, $84.1 \%$ and $0.15 \%$ for

42_11) were estimated in CheckM ${ }^{62}$. For comparison, Halanaerobium hydrogeniformans (txid: 656519), Anaerobaculum hydrogeniformans (txid: 592015), and Anaerobaculum mobile (txid: 891968) were run in BlastKOALA (family_eukaryotes+genus_prokaryotes) and the results are also included in Supplementary Data 2.

\section{Figure Legends:}

Figure 1. Schematic of the WL pathway reactions, and their relationships with other methylotrophy modules. In the $\mathrm{H}_{4}$ MPT methyl branch, Fwd denotes both the tungsten (Fwd) or molybdenum (Fmd) formylmethanofuran dehydrogenase complex (Fwd/FmdABCD). Ftr is the formylmethanofuran:tetrahydromethanopterin formyltransferase. Mch is the methenyltetrahydromethanopterin cyclohydrolase. Mtd/Hmd denote F420-dependent methylenetetrahydromethanopterin dehydrogenase and $\mathrm{H}_{2}$-forming methylenetetrahydromethanopterin dehydrogenase, respectively. bMtd refers to the bacterial methylene tetrahydromethanopterin dehydrogenase $(\mathrm{Mtd} \mathrm{A} / \mathrm{B} / \mathrm{C}$, which denote homologous enzyme classes, not subunits) to avoid confusion with the non-homologous archaeal Mtd. Mer is the methylenetetrahydromethanopterin reductase. Fhc stands for the bacterial enzyme complex composed of FwdABC+Ftr. 
The dashed arrow in the $\mathrm{H}_{4} \mathrm{~F}$ methyl branch indicates that spontaneous condensation of formaldehyde ( $\mathrm{HCHO}$ ) with $\mathrm{H}_{4} \mathrm{~F}$ can occur but is a very minor contribution to assimilatory fluxes ${ }^{26}$. Blue arrows denote reactions occurring in Archaea. Green arrows denote reactions occurring in methylotrophs. The glutathione (GSH)-dependent oxidation and ribulose monophosphate (RuMP) cycle are alternative pathways of formaldehyde assimilation found in methylotrophs, sometimes coexisting with the $\mathrm{H}_{4} \mathrm{MPT}$ branch. Note that throughout the text, unless specifically noted, MF and $\mathrm{H}_{4} \mathrm{MPT}$ are used as umbrella terms for all of their analogs which are produced through the same fundamental biosynthetic pathways, including methanofurans a-e, methylofuran, dephosphotetrahydromethanopterin, and tetrahydrosarcinapterin. See also Supplementary Figures 9 and 10 and their legends for details on MF and $\mathrm{H}_{4}$ MPT biosynthetic pathways.

Figure 2. (a) Schematic comparison of the length of the branch (average number of substitutions per site) separating Archaea and Bacteria in the phylogeny based on a concatenation of the three Fwd subunits common to Bacteria and Archaea (FwdABC) according to the taxonomic sampling available in 1999, 2004, and this study; (b) Bayesian phylogeny based on FwdABC (337 sequences and 859 aa). The phylogeny was inferred in PhyloBayes ${ }^{58}$ under the $\mathrm{CAT}+\mathrm{GTR}+\Gamma 4$ model. Values at nodes are posterior probabilities. Black circles denote branches with posterior probability of at least 0.95 . Scale bar represents the average number of substitutions per site. Some clades are collapsed, for clarity (the full tree is provided in Supplementary Figure S8). The tree is rooted according to one of the three possible roots for the Archaea, namely that between Euryarchaeota and TACK-Asgard, but bacteria emerge from within the archaeal radiation under all alternative rootings (see main text). The placement of 
Persephonearchaea, Theionarchaea and Altiarchaeales with respect to the root has not been yet specifically tested $^{18}$. For simplicity, the molybdenum-containing homolog (Fmd, or secondary) as well as additional divergent Fwd homologs (here referred to as "tertiary") present in Archaea (Supplementary Data 1) were analyzed separately and are presented in Supplementary Figure 22. W1-2 and W3-4 refer to the primary and secondary tungsten-containing Fwd clusters of Methanomicrobiales based on range of distribution (Supplementary Data 1); (c) Co-localization of $\mathrm{H}_{4} \mathrm{MPT}$ branch and cofactor biosynthesis genes. Gene order is mostly conserved (FwdDBAFtr-FwdC-Mch) between the uncultured archaeal and bacterial lineages close to the putative transfer event. The gene-to-biosynthetic reaction correspondence is noted with reaction numbers (H1 to H15 and M1 to M7/M7') and given in the table on the right for brevity. These reactions are presented in detail in Supplementary Figure $9 \& 10$. "O" is abbreviation for "ORF". Slashes indicate a contig edge. Figure was produced in $\mathrm{GeneSpy}^{50}$ by comparing a region of $10 \mathrm{kbp}$ centered around FwdA (or Mch when two sub-clusters existed in separate contigs).

Figure 3. Scenario for the origin and evolution of the $\mathrm{H}_{4} \mathrm{MPT}$ methyl branch in Bacteria. Colored circles denote the major events during the assembly of methylotrophy in Bacteria, as discussed in the main text. For simplicity the MF and H4MPT cofactor biosynthesis pathways are not shown, but they follow the same evolutionary history as the H4MPT branch (transferred at the red circle). Black circle: Initial configuration of the WL pathway in the LACA with a carbonyl and an $\mathrm{H}_{4} \mathrm{MPT}$ branch. Red circle: the $\mathrm{H}_{4} \mathrm{MPT}$ methyl branch is transferred from Archaea to Bacteria (FwdABCD, Ftr, Mch, and possibly bMtd). Fermentation products can enter the pathway through serine hydroxymethyltransferase and the Glycine Cleavage System. Green circle: Fae appears in Bacteria (possibly also transferred from Archaea). Methylated compounds 
can now enter the pathway through formaldehyde. Orange circle: FwdD is lost. FwdABC become associated with Ftr (Fhc complex), forcing the reaction into the $\mathrm{H}_{4} \mathrm{~F}$ branch. $\mathrm{HCHO}$, formaldehyde; $\mathrm{HCOOH}$, formate; $\mathrm{CH}_{3}$, methyl-; $\mathrm{CH}_{2^{-}}$, methylene--

Figure 4. Metabolic prediction of Limitata with main biochemical reactions. The reconstruction is derived from bacterium GBS-1 (Supplementary Data 2). The metabolism of bacterium 42_11 is almost identical, but was not used here, as the genome is incomplete. Apart from the GCS (see text for discussion), the $\mathrm{H}_{4} \mathrm{MPT}$ methyl branch can be also entered through other reactions (not shown), for instance at methylene- $\mathrm{H}_{4} \mathrm{MPT} / \mathrm{H}_{4} \mathrm{~F}$ through thymidylate synthase (ThyX) when converting dTMP to dUMP (in pyrimidine metabolism), through dehydropantoate hydroxymethyltransferase from 2-dehydropantoate (in pantothenate and CoA biosynthesis), or by diverting methionine into homocysteine and methyl- $\mathrm{H}_{4} \mathrm{MPT} / \mathrm{H}_{4} \mathrm{~F}$ (homocysteine methyltransferase) which is oxidized to methylene- $\mathrm{H}_{4} \mathrm{MPT} / \mathrm{H}_{4} \mathrm{~F}$ (methylene- $\mathrm{H}_{4} \mathrm{~F}$ reductase, not present in Limitata but in Halanaerobium). However, all these alternatives require intermediates of biosynthetic pathways, making it unlikely that they are consistently used as $\mathrm{H}_{4} \mathrm{MPT}$ branch input beyond some contribution during nucleotide or amino acid catabolism.

\section{References}

1. Singh, B. K., Bardgett, R. D., Smith, P. \& Reay, D. S. Microorganisms and climate change: terrestrial feedbacks and mitigation options. Nat. Rev. Microbiol. 8, 779-790 (2010).

2. Chistoserdova, L. \& Kalyuzhnaya, M. G. Current Trends in Methylotrophy. Trends Microbiol. 26, 703-714 (2018). 
3. Borrel, G. et al. Wide diversity of methane and short-chain alkane metabolisms in uncultured archaea. Nat. Microbiol. 4, 603-613 (2019).

4. Chistoserdova, L. et al. The enigmatic planctomycetes may hold a key to the origins of methanogenesis and methylotrophy. Mol. Biol. Evol. 21, 1234-1241 (2004).

5. Chistoserdova, L. Wide distribution of genes for tetrahydromethanopterin/methanofuranlinked $\mathrm{C} 1$ transfer reactions argues for their presence in the common ancestor of bacteria and archaea. Frontiers in Microbiology 7, 1425 (2016).

6. Weiss, M. C. et al. The physiology and habitat of the last universal common ancestor. Nat. Microbiol. 1, 16116 (2016).

7. Borrel, G., Adam, P. S. \& Gribaldo, S. Methanogenesis and the Wood-Ljungdahl Pathway: An Ancient, Versatile, and Fragile Association. Genome Biol. Evol. 8, 17061711 (2016).

8. Fuchs, G. Alternative pathways of carbon dioxide fixation: insights into the early evolution of life? Annu. Rev. Microbiol. 65, 631-658 (2011).

9. Laso-Pérez, R. et al. Thermophilic archaea activate butane via alkyl-coenzyme M formation. Nature 539, 396-401 (2016).

10. Chistoserdova, L. Modularity of methylotrophy, revisited. Environmental Microbiology 13, 2603-2622 (2011).

11. Chistoserdova, L., Vorholt, J. A., Thauer, R. K. \& Lidstrom, M. E. C1 transfer enzymes and coenzymes linking methylotrophic bacteria and methanogenic archaea. Science (80-. ). 281, 99-102 (1998).

12. Vorholt, J. A., Chistoserdova, L., Stolyar, S. M., Thauer, R. K. \& Lidstrom, M. E. Distribution of tetrahydromethanopterin-dependent enzymes in methylotrophic bacteria 
and phylogeny of methenyl tetrahydromethanopterin cyclohydrolases. J. Bacteriol. 181, 5750-5757 (1999).

13. Bauer, M. et al. Archaea-like genes for C1-transfer enzymes in Planctomycetes: Phylogenetic implications of their unexpected presence in this phylum. J. Mol. Evol. 59, $571-586$ (2004).

14. Kalyuzhnaya, M. G. et al. Analysis of gene islands involved in methanopterin-linked C1 transfer reactions reveals new functions and provides evolutionary insights. J. Bacteriol. 187, 4607-4614 (2005).

15. Butterfield, C. N. et al. Proteogenomic analyses indicate bacterial methylotrophy and archaeal heterotrophy are prevalent below the grass root zone. PeerJ 4, e2687 (2016).

16. Ettwig, K. F. et al. Nitrite-driven anaerobic methane oxidation by oxygenic bacteria. Nature 464, 543-548 (2010).

17. Raymann, K., Brochier-Armanet, C. \& Gribaldo, S. The two-domain tree of life is linked to a new root for the Archaea. Proc. Natl. Acad. Sci. 112, 6670-6675 (2015).

18. Adam, P. S., Borrel, G., Brochier-Armanet, C. \& Gribaldo, S. The growing tree of Archaea: New perspectives on their diversity, evolution and ecology. ISME J. 11, 24072425 (2017).

19. Ramamoorthy, S. et al. Desulfosporosinus lacus sp. nov., a sulfate-reducing bacterium isolated from pristine freshwater lake sediments. Int. J. Syst. Evol. Microbiol. 56, 27292736 (2006).

20. Davidova, I. A. et al. Dethiosulfatarculus sandiegensis gen. nov., sp. nov., isolated from a methanogenic paraffindegrading enrichment culture and emended description of the family Desulfarculaceae. Int. J. Syst. Evol. Microbiol. 66, 1242-1248 (2016). 
21. Adam, P. S., Borrel, G. \& Gribaldo, S. Evolutionary history of carbon monoxide dehydrogenase/acetyl-CoA synthase, one of the oldest enzymatic complexes. Proc. Natl. Acad. Sci. 115, E5837 (2018).

22. Sousa, F. L. \& Martin, W. F. Biochemical fossils of the ancient transition from geoenergetics to bioenergetics in prokaryotic one carbon compound metabolism. Biochim. Biophys. Acta - Bioenerg. 1837, 964-981 (2014).

23. Chistoserdova, L., Rasche, M. E. \& Lidstrom, M. E. Novel Dephosphotetrahydromethanopterin Biosynthesis Genes Discovered via Mutagenesis in Methylobacterium extorquens AM1. J. Bacteriol. 187, 2508-2512 (2005).

24. Pomper, B. K., Saurel, O., Milon, A. \& Vorholt, J. A. Generation of formate by the formyltransferase/hydrolase complex (Fhc) from Methylobacterium extorquens AM1. FEBS Lett. 523, 133-137 (2002).

25. Wagner, T., Ermler, U. \& Shima, S. The methanogenic CO2 reducing-and-fixing enzyme is bifunctional and contains 46 [4Fe-4S] clusters. Science (80-. ). 354, 114-117 (2016).

26. Crowther, G. J., Kosaly, G. \& Lidstrom, M. E. Formate as the main branch point for methylotrophic metabolism in Methylobacterium extorquens AM1. J. Bacteriol. 190, 5057-5062 (2008).

27. Bar-Even, A., Noor, E. \& Milo, R. A survey of carbon fixation pathways through a quantitative lens. Journal of Experimental Botany 63, 2325-2342 (2012).

28. Figueroa, I. A. et al. Metagenomics-guided analysis of microbial chemolithoautotrophic phosphite oxidation yields evidence of a seventh natural $\mathrm{CO}_{2}$ fixation pathway. Proc. Natl. Acad. Sci. 201715549 (2017). doi:10.1073/pnas.1715549114

29. Vorholt, J. A., Marx, C. J., Lidstrom, M. E. \& Thauer, R. K. Novel formaldehyde- 
activating enzyme in Methylobacterium extorquens AM1 required for growth on methanol. J. Bacteriol. 182, 6645-6650 (2000).

30. Lin, Z. \& Sparling, R. Investigation of serine hydroxymethyltransferase in methanogens. Can. J. Microbiol. 44, 652-656 (1998).

31. Schwartz, E., Fritsch, J. \& Friedrich, B. in The Prokaryotes 119-199 (2013). doi:10.1007/978-3-642-30141-4_65

32. Begemann, M. B., Mormile, M. R., Sitton, O. C., Wall, J. D. \& Elias, D. A. A streamlined strategy for biohydrogen production with Halanaerobium hydrogeniformans, an alkaliphilic bacterium. Front. Microbiol. 3, 93 (2012).

33. Maune, M. W. \& Tanner, R. S. Description of Anaerobaculum hydrogeniformans sp. nov., an anaerobe that produces hydrogen from glucose, and emended description of the genus Anaerobaculum. Int. J. Syst. Evol. Microbiol. 62, 832-838 (2012).

34. Liang, R., Grizzle, R. S., Duncan, K. E., McInerney, M. J. \& Suflita, J. M. Roles of thermophilic thiosulfate-reducing bacteria and methanogenic archaea in the biocorrosion of oil pipelines. Front. Microbiol. 5, 89 (2014).

35. Kasting, J. F. Methane and climate during the Precambrian era. Precambrian Res. 137, $119-129$ (2005).

36. Ueno, Y., Yamada, K., Yoshida, N., Maruyama, S. \& Isozaki, Y. Evidence from fluid inclusions for microbial methanogenesis in the early Archaean era. Nature 440, 516-519 (2006).

37. Slotznick, S. P. \& Fischer, W. W. Examining Archean methanotrophy. Earth Planet. Sci. Lett. 441, 52-59 (2016).

38. Summons, R. E., Jahnke, L. L. \& Roksandic, Z. Carbon isotopic fractionation in lipids 
from methanotrophic bacteria: Relevance for interpretation of the geochemical record of biomarkers. Geochim. Cosmochim. Acta 58, 2853-2863 (1994).

39. Jahnke, L. L., Summons, R. E., Hope, J. M. \& Des Marais, D. J. Carbon isotopic fractionation in lipids from methanotrophic bacteria II: The effects of physiology and environmental parameters on the biosynthesis and isotopic signatures of biomarkers. Geochim. Cosmochim. Acta 63, 79-93 (1999).

40. Battistuzzi, F. U. \& Hedges, S. B. A major clade of prokaryotes with ancient adaptations to life on land. Mol. Biol. Evol. 26, 335-343 (2009).

41. Haqq-Misra, J. D., Domagal-Goldman, S. D., Kasting, P. J. \& Kasting, J. F. A revised, hazy methane greenhouse for the Archean Earth. Astrobiology 8, 1127-1137 (2008).

42. Konhauser, K. O. et al. Oceanic nickel depletion and a methanogen famine before the Great Oxidation Event. Nature 458, 750-753 (2009).

43. Catling, D. C., Claire, M. W. \& Zahnle, K. J. Anaerobic methanotrophy and the rise of atmospheric oxygen. Philos. Trans. R. Soc. A Math. Phys. Eng. Sci. 365, 1867-1888 (2007).

44. Daines, S. J. \& Lenton, T. M. The effect of widespread early aerobic marine ecosystems on methane cycling and the Great Oxidation. Earth Planet. Sci. Lett. 434, 42-51 (2016).

45. Markowitz, V. M. et al. IMG: the integrated microbial genomes database and comparative analysis system. Nucleic Acids Res. 40, D115-D122 (2012).

46. Wrighton, K. C. et al. Fermentation, hydrogen, and sulfur metabolism in multiple uncultivated bacterial phyla. Science (80-. ). 337, 1661-1665 (2012).

47. Hyatt, D. et al. Prodigal: Prokaryotic gene recognition and translation initiation site identification. BMC Bioinformatics 11, 119 (2010). 
48. Kanehisa, M., Furumichi, M., Tanabe, M., Sato, Y. \& Morishima, K. KEGG: New perspectives on genomes, pathways, diseases and drugs. Nucleic Acids Res. 45, D353D361 (2017).

49. Johnson, L. S., Eddy, S. R. \& Portugaly, E. Hidden Markov model speed heuristic and iterative HMM search procedure. BMC Bioinformatics 11, 431 (2010).

50. Garcia, P. S., Jauffrit, F., Grangeasse, C. \& Brochier-Armanet, C. GeneSpy, a userfriendly and flexible genomic context visualizer. Bioinformatics 35, 329-331 (2019).

51. Abby, S. S., Néron, B., Ménager, H., Touchon, M. \& Rocha, E. P. C. MacSyFinder: A program to mine genomes for molecular systems with an application to CRISPR-Cas systems. PLoS One 9, e110726 (2014).

52. Edgar, R. C. MUSCLE: Multiple sequence alignment with high accuracy and high throughput. Nucleic Acids Res. 32, 1792-1797 (2004).

53. Criscuolo, A. \& Gribaldo, S. BMGE (Block Mapping and Gathering with Entropy): A new software for selection of phylogenetic informative regions from multiple sequence alignments. BMC Evol. Biol. 10, 210 (2010).

54. Nguyen, L. T., Schmidt, H. A., Von Haeseler, A. \& Minh, B. Q. IQ-TREE: A fast and effective stochastic algorithm for estimating maximum-likelihood phylogenies. Mol. Biol. Evol. 32, 268-274 (2015).

55. Kobert, K., Salichos, L., Rokas, A. \& Stamatakis, A. Computing the Internode Certainty and Related Measures from Partial Gene Trees. Mol. Biol. Evol. 33, 1606-1617 (2016).

56. Stamatakis, A. RAxML-VI-HPC: Maximum likelihood-based phylogenetic analyses with thousands of taxa and mixed models. Bioinformatics 22, 2688-2690 (2006).

57. Hoang, D. T., Chernomor, O., von Haeseler, A., Minh, B. Q. \& Vinh, L. S. UFBoot2: 
improving the ultrafast bootstrap approximation. Mol. Biol. Evol. 35, 518-522 (2017).

58. Lartillot, N., Lepage, T. \& Blanquart, S. PhyloBayes 3: A Bayesian software package for phylogenetic reconstruction and molecular dating. Bioinformatics 25, 2286-2288 (2009).

59. Kanehisa, M., Sato, Y. \& Morishima, K. BlastKOALA and GhostKOALA: KEGG Tools for Functional Characterization of Genome and Metagenome Sequences. Journal of Molecular Biology 428, 726-731 (2016).

60. Altschul, S. F., Gish, W., Miller, W., Myers, E. W. \& Lipman, D. J. Basic local alignment search tool. J. Mol. Biol. 215, 403-410 (1990).

61. Overbeek, R. et al. The SEED and the Rapid Annotation of microbial genomes using Subsystems Technology (RAST). Nucleic Acids Res. 42, D206-D214 (2014).

62. Parks, D. H., Imelfort, M., Skennerton, C. T., Hugenholtz, P. \& Tyson, G. W. CheckM: Assessing the quality of microbial genomes recovered from isolates, single cells, and metagenomes. Genome Res. 25, 1043-1055 (2015).

Author notes: S.G. and P.S.A. designed research; P.S.A. performed all phylogenomic analyses; P.S.A. and G.B. performed genome synteny and metabolic reconstruction analyses; P.S.A., G.B., and S.G. analyzed the data and wrote the paper.

Data availability: All raw data (HMM profiles, initial and trimmed alignments, full trees in Newick format) are available in Supplementary Data 4.

Acknowledgments: P.S.A. is supported by a PhD fellowship from Paris Diderot University and by funds from the PhD Programme "Frontières du Vivant (FdV)-Programme Bettencourt." G.B. 
acknowledges support from the Institut Pasteur through a Roux-Cantarini fellowship. S.G.

acknowledges funding from the French National Agency for Research Grant ArchEvol (ANR-

16-CE02-0005-01).

Competing interests: The authors declare no competing interests.

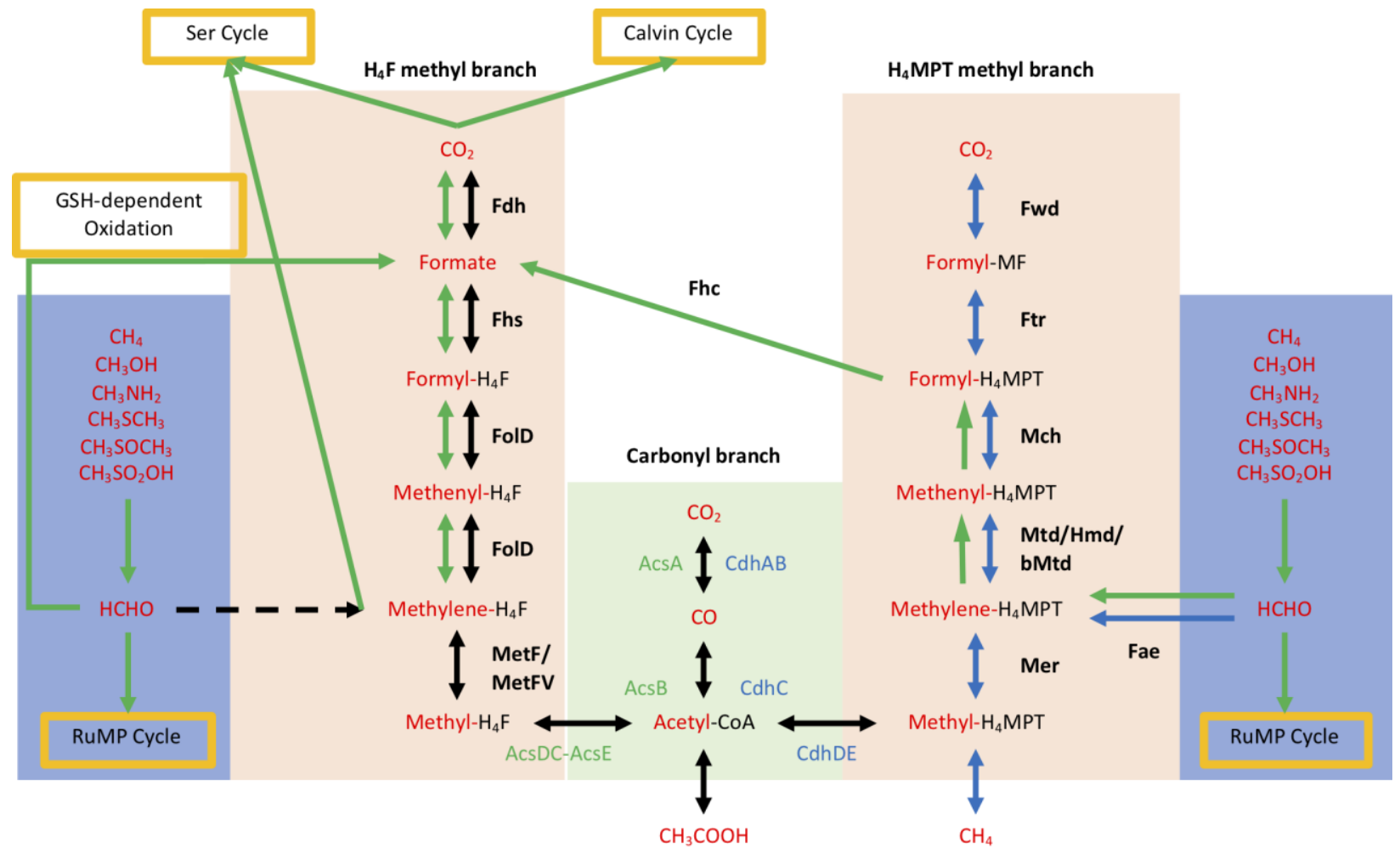

Figure 1 


\section{a}

b

$\beta, \gamma$-Proteobacteria
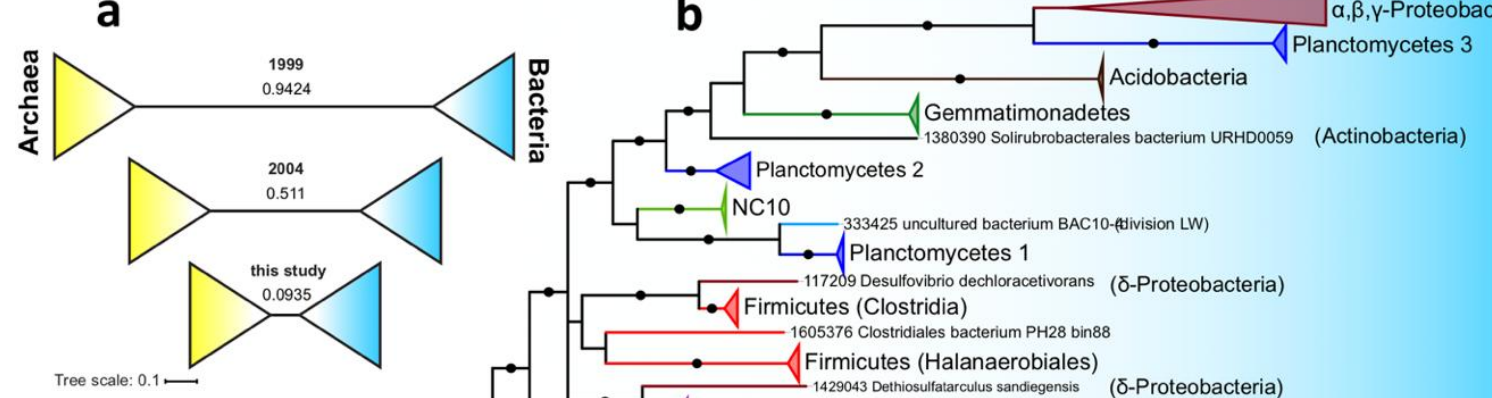

DPlanctomycetes 2

C

Tree scale: 0.1

117209 Desulfovibrio dechloracetivorans
(D-Proteobacteria) $\rightarrow \quad$ Limitata

1775755 Hadesarchaea archaeon DG-33-1
(Hadesarchaea)
1775754 Hadesarchaea archaeon DG-33
P Persephonarchaea 1

Bacteria

(a)

Altiarchaeales

Bathyarchaeota 1

1538547 Lokiarchaeum sp. GC14 75(Lokiarchaeota)

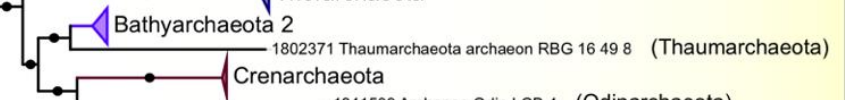

TACK-Asgard

- 1841599 Archaeon Odin LCB 4 (Odinarchaeota)

- Persephonarchaea 2

$\longrightarrow$ MF Biosynth. $\Rightarrow$ H4MPT biosynth. $\Rightarrow$ putative H4MPT biosynth.

\begin{tabular}{|c|c|c|c|c|}
\hline & \multirow{4}{*}{$\begin{array}{r}\text { Acidobacteria bac 13_1_20CM_2_68_7 (Acidobacteria) } \\
\text { Gemmatimonadetes bac 13_1_40CM_69_22 (Gemmatimonadetes) }\end{array}$} & \multirow{13}{*}{ 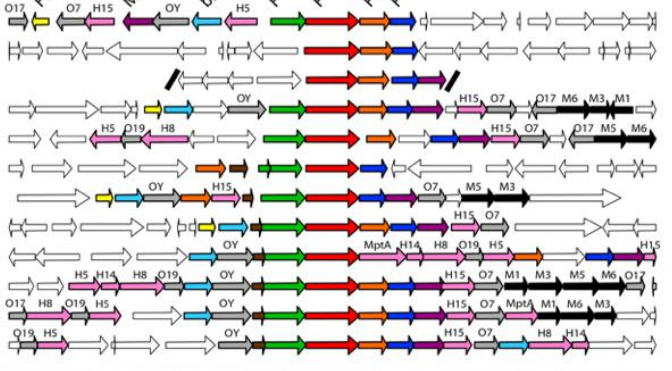 } & Reaction & Enzyme/Orf \\
\hline & & & \multicolumn{2}{|c|}{ MF biosyntesis } \\
\hline & & & M1 & MfnB/Orf22 \\
\hline & & & M2 & MfnC \\
\hline & Paludisphaera borealis (Planctomycetes 2) & & M3 & MfnE/Orf21 \\
\hline & Planctomycetaceae bacterium TMED240 (Planctomycetes 1) & & M4 & MfnA \\
\hline & Division LW bacterium BAC10-4 (division LW) & & M5 & MfnD/Orf1 \\
\hline & \multirow{6}{*}{$\begin{array}{r}\text { Acetomicrobium hydrogeniformans ATCC BAA-1850 (Synergistetes) } \\
\text { Desulfosporosinus lacus DSM } 15449 \text { (Clostridia) } \\
\text { Halanaerobium hydrogeniformans (Halanerobiales) } \\
\text { Limitata bacterium GBS-1 (Limitata) }\end{array}$} & & M6 & MfnF/Orf9 \\
\hline & & & $\mathrm{M} 7 / \mathrm{M7}^{\prime}$ & $?$ \\
\hline & & & \multicolumn{2}{|c|}{ HaMPT biosynthesis } \\
\hline & & & H1 & MptD \\
\hline & & & H2 & MJ0619 \\
\hline & & & H3 & MptE \\
\hline & MSBL1 archaeon SCGC-AAA259E19 (Persephonarchaea 1) & & H4 & MJ0807 \\
\hline & Hadesarchaea archaeon DG-33-1 (Hadesarchaea) & & H5 & MptG \\
\hline & Theionarchaea archaeon DG-70 (Theionarchaea) & $\Rightarrow$ & H6 & OrfY (MJ0776)? \\
\hline & & $\Leftrightarrow \Leftrightarrow \Rightarrow \Rightarrow$ & H7 & MJ0929 \\
\hline & Altiarchaeales archaeaon SM1-MSI (Altiarchaea, Fwd) & & $\mathrm{H} 8$ & MptH/Orf20 \\
\hline & & & H9 & Orf17? \\
\hline & Ca. Altiarchaeales archaeon IMC4 (Altiarchaea, Fmd) & 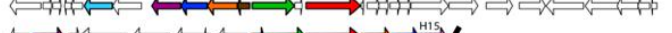 & $\mathrm{H} 10$ & $?$ \\
\hline & Miscell. Crenarchaeota group-15 arc DG-45 (Bathyarchaeota 1) & $\Leftrightarrow \Rightarrow+2 \Leftrightarrow$ & H11 & Orf7? \\
\hline & Ca. Thorarchaeota archaeon SMTZ1-83 (Thorarchaeota) & 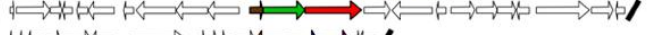 & H12 & Orf?? \\
\hline & & 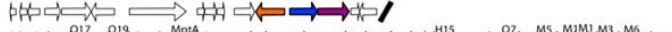 & $\mathrm{H} 13$ & $?$ \\
\hline & Vulcanisaeta sp. JCM 14467 (Crenarchaeota) & 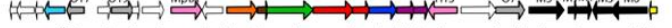 & H14 & DmrX \\
\hline & Ca. Bathyarchaeota archaeon BA1 (Bathyarchaeota 2) & $\Rightarrow \Rightarrow \Leftrightarrow \Leftrightarrow \Rightarrow \Rightarrow \Leftrightarrow \Leftrightarrow$ & H15 & MptN/Orf5 \\
\hline & MSBL1 archaeon SCGC-AAA259E22 (Persephonarchaea 2) & & $\mathrm{H}$ ? & Orf19 \\
\hline
\end{tabular}

Figure 2 


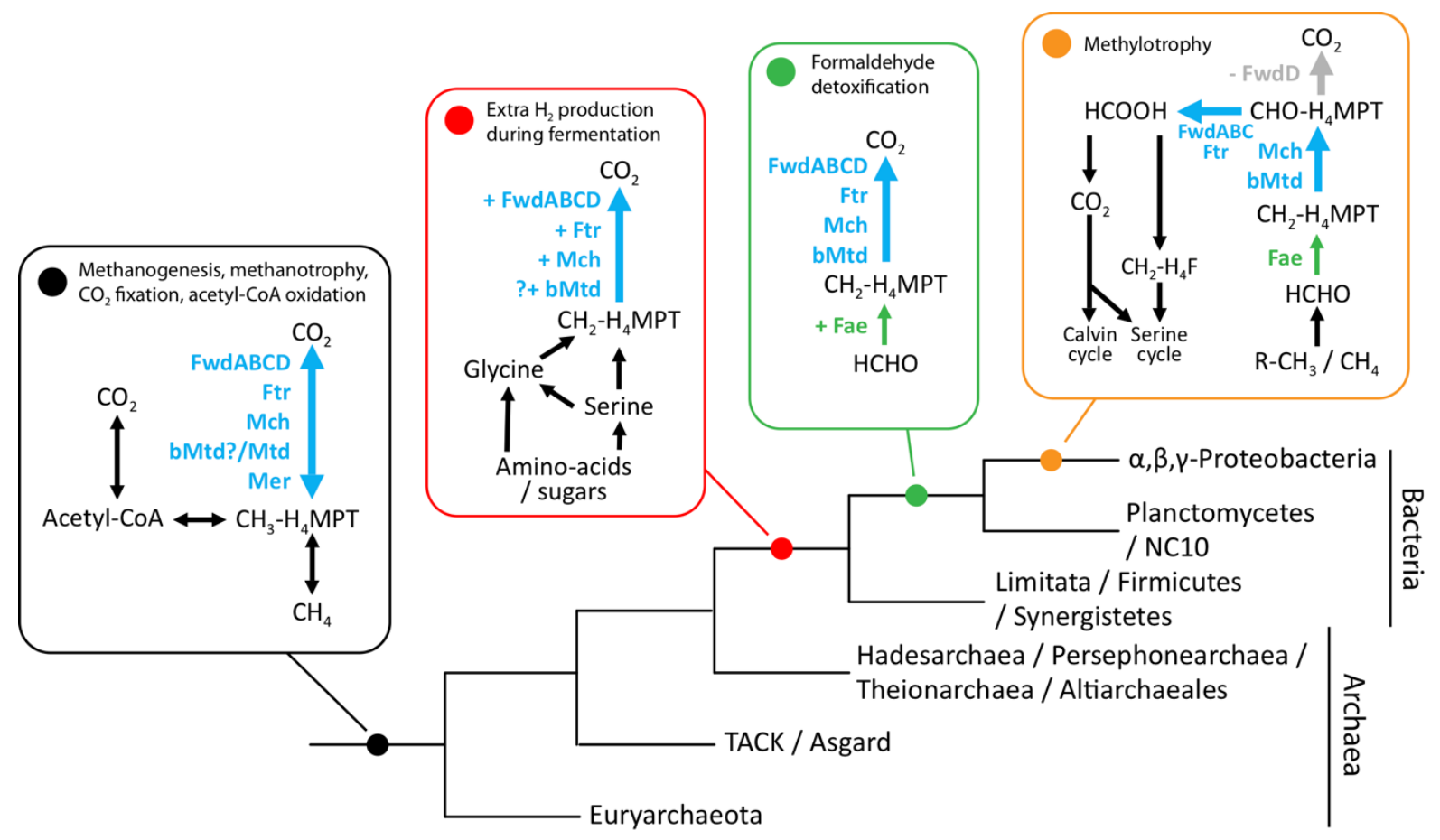

Figure 3 


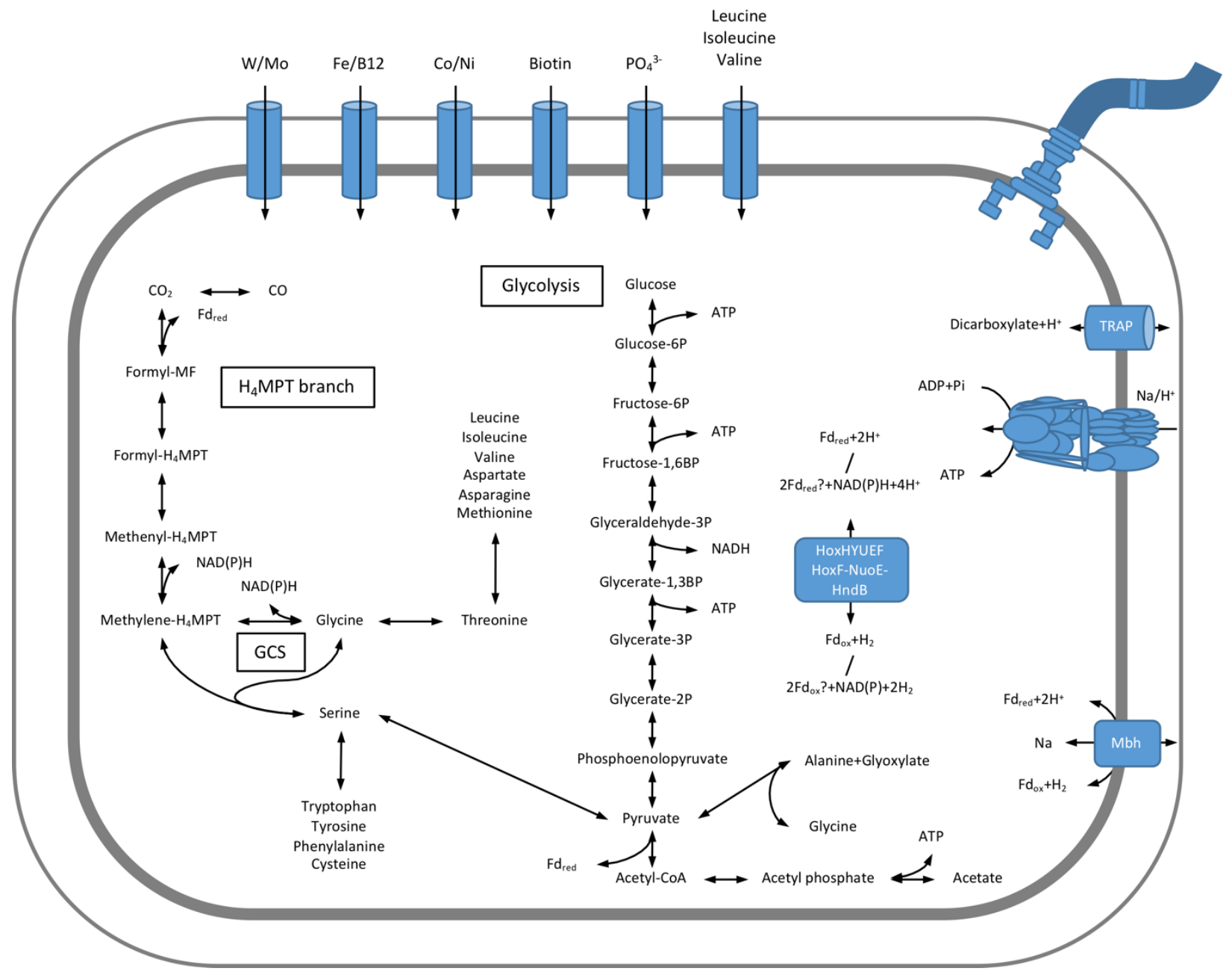

Figure 4 